\title{
Elastic and absorption cross sections for electron-hydroxyl radical collisions
}

\author{
A. M. C. Sobrinho \\ Departamento de Física, UFSCar 13565-905, São Carlos, SP, Brazil \\ and Departamento de Física de Estado Sólido, UFBA 40170-290, Salvador, BA, Brazil \\ N. B. H. Lozano and M.-T. Lee \\ Departamento de Química, UFSCar 13565-905 São Carlos, SP, Brazil \\ (Received 30 March 2004; published 28 September 2004)
}

\begin{abstract}
In this work, we report a theoretical study on electron collisions with $\mathrm{OH}$ radicals in the low and intermediate energy ranges. Calculated elastic differential, integral, and momentum-transfer cross sections as well as grand-total (elastic + inelastic) and total absorption cross sections for electron-OH collisions are reported in the 1-500-eV range. A complex optical potential composed by static, exchange, correlation-polarization plus absorption contributions, derived from a fully molecular wave function, is used to describe the interaction dynamics. The Schwinger variational iterative method combined with the distorted-wave approximation is applied to calculate scattering amplitudes. Present calculated results are compared with the existing data for electron-OH scattering in the literature. Also, comparison made between our calculated cross sections for elastic scattering with the theoretical and experimental results for electron- $\mathrm{H}_{2} \mathrm{O}$ collisions has revealed remarkable similarity even at incident energies as low as $2 \mathrm{eV}$.
\end{abstract}

DOI: 10.1103/PhysRevA.70.032717

PACS number(s): $34.80 . \mathrm{Bm}$

\section{INTRODUCTION}

Cross sections for electron scattering from a variety of molecules and free radicals are demanded in different fields of the pure and applied sciences [1-3]. In particular, interest in electron collisions with reactive radicals has grown recently [4-8], in view of their important role in physical and chemical processes involved in a number of applications such as lasers [9], gas discharges, plasmas [10], etc., as well as in the atmospheric and astrophysical studies [11]. The hydroxyl $(\mathrm{OH})$ is one of such radicals. The $\mathrm{OH}$ radicals can be produced in Earth's atmosphere by vuv photolysis of water [12]. Due to its high reactivity, $\mathrm{OH}$ is an important reaction intermediate in the atmospheric chemistry [13-16]. The $\mathrm{OH}$ radicals have also been identified in the extreme carbon star IRC +10216 [17] as well as in the comets Hale-Bopp [18] and C/1999 HI(Lee) [19]. Moreover, OH plays an important role in biology and medicine. It is well known that the nuclear radiation that penetrates into the human body may cause several types of cancer. The $\mathrm{OH}$ radicals are pointed out as a precursor of this disease because it may cause damages in cells [20-23]. In the human body, $\mathrm{OH}$ is formed by the interaction of the water molecule and secondary electrons with appreciable kinetic energy, which are produced by nuclear radiation [24].

Because of its relevant application in a number of fields, the knowledge of the electron-OH collisional dynamics is certainly of interest. However, there is a lack of either experimental or theoretical studies on such matter in the literature. Due to its high chemical reactivity, it would be very difficult to generate a $\mathrm{OH}$ radical beam to be interacted with electron beam, thus experimental studies on electron-OH collisions would be a very hard task. To our knowledge, there is only one article [25] that reports the measurement of total ionization cross sections (TICS's) for electron-OH collisions. Theoretically, studies for electron-OH scattering are equally scarce. Low-energy electron scattering from the ground electron state $\left({ }^{2} \Pi\right)$ of $\mathrm{OH}$ was investigated by Chen and Morgan [26] using the $R$-matrix method. In that work, they reported calculated cross sections for the $0 \rightarrow 1$ vibrational transition of $\mathrm{OH}$ by electron impact in the $0-3-\mathrm{eV}$ energy range as well as the differential cross sections (DCS's) for elastic $e^{-}-\mathrm{OH}$ scattering at a single incident energy of $1.58 \mathrm{eV}$. Also, Joshipura et al. [5] have calculated total cross sections (TCS's) and total absorption cross sections (TACS's), and estimated TICS's for electron-OH collisions inthe 10-2000-eV range. A complex optical potential for electron-atom interaction combined with the additivity rule was used in their calculations. Although this method may provide reliable cross sections for incident energies above $100 \mathrm{eV}$, it is expected to fail at lower energies. In this work, we present a theoretical study on electron-OH scattering covering a wide incident energy range. More specifically, DCS's, integral (ICS's) and momentum transfer (MTCS's) cross sections for elastic $e^{-}-\mathrm{OH}$ scattering as well as TCS's and TACS's in the $1-500-\mathrm{eV}$ energy range are calculated and reported. The present study made use of a complex optical potential to represent the electron-radical interaction, whereas a combination of the Schwinger variational iterative method (SVIM) [27,28] and the distorted-wave approximation (DWA) [29-31] is used to solve the scattering equations. This procedure has already been applied to treat electron scattering by a number of molecules [32-35] and radicals [6-8] and has provided reliable DCS's, ICS's, and MTCS's over a wide energy range.

The organization of this paper is as follows. In Sec. II, we describe briefly the theory used and also some details of the calculation. In Sec. III we present our calculated results. 


\section{THEORY AND CALCULATION}

Details of the SVIM [27,28] and the DWA [29-31] have already been presented previously, and will only be outlined here. Within the fixed-nuclei framework, the electronmolecule scattering dynamics is represented by a complex optical potential,

$$
V_{\text {opt }}(\vec{r})=V^{S E P}(\vec{r})+i V_{a b}(\vec{r}),
$$

where the $V^{S E P}$ is the real part of the interaction potential composed by the static $\left(V_{s t}\right)$, the exchange $\left(V_{e x}\right)$, and the correlation-polarization contributions $\left(V_{c p}\right)$ whereas $V_{a b}$ is the absorption potential. In our calculation, $V_{s t}$ and $V_{e x}$ are derived exactly from a restricted open-shell Hartree-Fock (ROHF) self-consistent-field (SCF) target wave function. A parameter-free model potential introduced by Padial and Norcross [36] is used to account for the correlationpolarization contributions. In this model, a short-range correlation potential between the scattering and target electrons is defined in an inner interaction region and a long-range polarization potential in an outer region. The first crossing of the correlation and polarization potential curves defines the inner and outer regions. The short-range correlation potential is derived using the target electronic density according to Eq. (9) of Padial and Norcross [36]. In addition, an asymptotic form of the polarization potential is used for the long-range electron-target interactions. The model potential of Padial and Norcross is in fact rather crude. Specifically, the use of the free-electron-gas approximation in the inner region may not reflect realistically the properties of targets. Also, the junction of the potentials calculated separately in the inner and outer regions is somehow arbitrary. Despite that, the simplicity of this model makes it quite useful in theoretical studies of electron scattering by atoms and molecules. Moreover, $V_{c p}$ generated by this model has the correct asymptotic form of polarization potential which is very interesting for low-energy electron-molecule scattering, due to the low penetration capacity of these electrons into targets. The application of this model requires dipole polarizabilities to generate the asymptotic form of $V_{c p}$. Since there is no experimental and/or theoretical values available in the literature for the $\mathrm{OH}$ radical, they were calculated in this work at $\mathrm{ROHF}$ level of approximation. The calculated values are $\alpha_{z z}=7.885$ a.u. and $\alpha_{x x}=5.942$ a.u. No cutoff or other adjusted parameters are needed in the calculation of $V_{c p}$.

Although the main features of the absorption effects are known, taking these effects into account in an $a b$ initio treatment of electron-molecule scattering is very difficult. Presently, despite the ab initio methods (e.g., $R$ matrix [37], Kohn variational [38], and Schwinger multichannel [39], etc.) are routinely applied to electron-molecule collisional studies, these applications are in general limited in the low incident energy range $(\leqslant 30 \mathrm{eV})$ where the absorption effects are absent or small. In order to treat the absorption effects, those methods of close-coupling nature would require all discrete and continuum open channels to be included in the openchannel $P$ space, which are still computationally unfeasible. In view of the difficulties, the use of the model absorption potential seems to be presently the only practical manner to account for absorption effects into electron-molecule scattering calculations. Several model absorption potentials have been proposed and used, but version 3 of the quasifree scattering model (QFSM) proposed by Staszewska et al. [40], and lately modified by Jain and Baluja [41], has shown to yield cross sections in better agreement when compared with experiments. We have chosen the latter to account for the absorption component of the electron-radical interaction potential. The absorption potential $V_{a b}$ in Eq. (1) is given by

$$
V_{a b}(\vec{r})=-\rho(\vec{r})\left(T_{L} / 2\right)^{1 / 2}\left(8 \pi / 5 k^{2} k_{F}^{3}\right) H\left(\alpha+\beta-k_{F}^{2}\right)(A+B+C),
$$

where

$$
\begin{gathered}
T_{L}=k^{2}-V^{S E P}, \\
A=5 k_{F}^{3} /\left(\alpha-k_{F}^{2}\right), \\
B=-k_{F}^{3}\left[5\left(k^{2}-\beta\right)+2 k_{F}^{2}\right] /\left(k^{2}-\beta\right)^{2},
\end{gathered}
$$

and

$$
C=2 H\left(\alpha+\beta-k^{2}\right) \frac{\left(\alpha+\beta-k^{2}\right)^{5 / 2}}{\left(k^{2}-\beta\right)^{2}} .
$$

In Eqs. (2)-(6), $k^{2}$ is the energy (in Rydbergs) of the incident electron, $k_{F}$ the Fermi momentum, and $\rho(\vec{r})$ the local electronic density of the target. $H(x)$ is a Heaviside function defined by $H(x)=1$ for $x \geqslant 0$ and $H(x)=0$ for $x<0$. In the modified QFSM version 3 [41],

$$
\alpha(\vec{r}, E)=k_{F}^{2}+2(2 \Delta-I)-V^{S E P},
$$

and

$$
\beta(\vec{r}, E)=k_{F}^{2}+2(I-\Delta)-V^{S E P},
$$

where $\Delta$ is the average excitation energy and $I$ is the ionization potential. In the original version of Staszewska et al., $V^{S E P}$ present in Eqs. (3), (7), and (8) are replaced by the static-exchange potential $V^{S E}$ in the calculation of the local velocity of the scattering electron. In the present study, the experimental first ionization potential $(13.17 \mathrm{eV})$ [42] is used as the average excitation energy as suggested by Jain and Baluja [41].

Since $\mathrm{OH}$ is an open-shell target, the coupling of the incident electron with the unpaired $1 \pi$ electron of the target leads to two spin-specific scattering channels, namely, the singlet $(\mathrm{S}=0)$ and triplet $(\mathrm{S}=1)$ couplings. The main difference between the singlet and triplet scattering channels would reflect on the treatment of the electron-exchange term in the potential operator. On the other hand, contributions such as $V_{s t}, V_{c p}$, and $V_{a b}$ are calculated in the present study using the target electronic density and some molecular properties such as ionization potential, dipole polarizability, etc. Thus they are not explicitly dependent on the spin couplings.

Further, the spin-specific Lippmann-Schwinger (LS) equation is solved using the SVIM. In principle, this scattering equation for elastic $e^{-}-\mathrm{OH}$ scattering should be solved with the full complex optical potential. Nevertheless, a tremendous computational effort would be required, particu- 
larly due to the large number of coupled equations involved, which makes such calculations practically prohibitive. On the other hand, our calculation has revealed that the magnitude of the imaginary part (absorption) of the optical potential is considerably smaller than its real counterpart. So, it can be treated as a perturbation. Therefore, in the present study, the LS scattering equations are solved using SVIM considering only the real part of the optical potential. In the SVIM calculations, the continuum wave functions are singlecenter expanded as

$$
\chi_{\vec{k}}^{ \pm, S}(\vec{r})=(2 / \pi)^{1 / 2} \sum_{l m} \frac{(i)^{l}}{k} \chi_{k l m}^{ \pm, S}(\vec{r}) Y_{l m}(\hat{k}),
$$

where the superscripts $(+)$ and $(-)$ denote the incoming-wave and outgoing-wave boundary conditions, respectively, $S$ is the total spin of the (electron + target) system, and $Y_{l m}(\hat{k})$ are the usual spherical harmonics. The absorption part of the $T$ matrix is calculated via the DWA as

$$
T_{a b s}=i\left\langle\chi_{f}^{-}\left|V_{a b}\right| \chi_{i}^{+}\right\rangle .
$$

In the present work, we have limited the partial-wave expansion of $T$-matrix elements up to $l_{\max }=40$ and $m_{\max }=17$. A Born-closure procedure is used to account for the contribution of higher partial-wave dipole components to scattering amplitudes. In order to avoid the divergent behavior of the DCS's in the forward direction, nuclear-rotational dynamics is treated explicitly.

The spin-specific rotational scattering amplitude is expressed as

$$
f_{j m_{j} \leftarrow j_{0} m_{j_{0}}}^{S}=\left\langle j m_{j}\left|f^{S}\right| j_{0} m_{j_{0}}\right\rangle,
$$

where $\left|j m_{j}\right\rangle$ are the rigid-rotor wave functions and $f^{(S)}$ the spin-specific fixed-nuclear electron scattering amplitude in the laboratory frame (LF). Accordingly, the spin-specific DCS's for the rotational excitation from an initial level $j_{0}$ to a final level $j$ is given by

$$
\left(\frac{d \sigma}{d \Omega}\right)^{S}\left(j \leftarrow j_{0}\right)=\frac{k_{f}}{k_{0}} \frac{1}{\left(2 j_{0}+1\right)} \sum_{m_{j} m_{j_{0}}}\left|f_{j m_{j} \leftarrow j_{0} m_{j_{0}}}\right|^{2},
$$

where $k_{f}$ and $k_{0}$ are the final and initial linear momenta of the scattering electron, respectively.

Moreover, the spin-specific rotationally unresolved DCS's for elastic $e^{-}-\mathrm{OH}$ scattering are calculated via a summation of rotationally resolved DCS's,

$$
\left(\frac{d \sigma}{d \Omega}\right)^{S}=\sum_{j=0}\left(\frac{d \sigma}{d \Omega}\right)^{S}\left(j \leftarrow j_{0}\right) .
$$

Finally, the spin-average DCS's for elastic $e^{-}-\mathrm{OH}$ scattering is calculated using the statistical weight for singlet $(1 / 4)$ and triplet (3/4) scattering channels, as

$$
\left(\frac{d \sigma}{d \Omega}\right)=\frac{1}{4}\left[3\left(\frac{d \sigma}{d \Omega}\right)^{S=1}+\left(\frac{d \sigma}{d \Omega}\right)^{S=0}\right] .
$$

In the present study, a standard basis set of Dunning [43] augmented by three $s(\alpha=0.05,0.02$, and 0.005$)$, one $p(\alpha$ $=0.04)$, and three $d(\alpha=1.7,0.85$, and 0.34) uncontracted

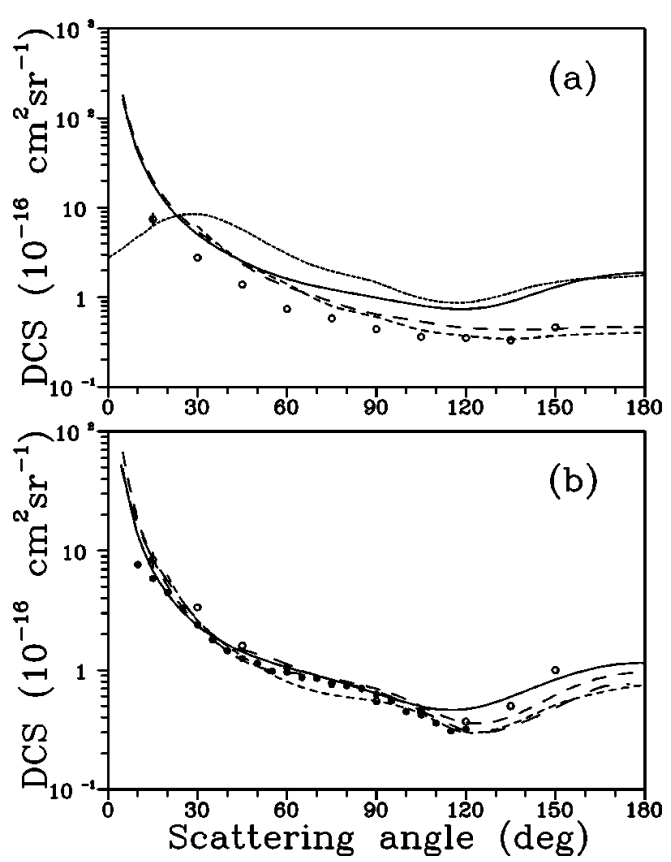

FIG. 1. DCS's for elastic $e^{-}-\mathrm{OH}$ scattering at (a) $2 \mathrm{eV}$ and (b) $6 \mathrm{eV}$. Solid line, present rotationally summed results; dotted line, the $R$-matrix DCS's at $1.58 \mathrm{eV}$; the calculated results for $e^{-}-\mathrm{H}_{2} \mathrm{O}$ scattering are of: short-dashed line, Rescigno and Lengsfield [46]; dashed line, Machado et al. [47]; long-dashed line, Gianturco [45]. The experimental results for $e^{-}-\mathrm{H}_{2} \mathrm{O}$ scattering are of: open circles, Shyn and Cho [52]; full circles, Johnstone and Newell [53]. The quoted experimental uncertainties of the DCS's vary from $9 \%$ to $15 \%$. Representative error bars of some selected data are also shown.

functions for oxygen atom and a Gaussian basis set of Huzinaga [44] augmented by three $p(\alpha=1.0,0.5$, and 0.1$)$ uncontracted functions for the hydrogen atom are used for the calculation of the SCF wave function of the target. At the experimental equilibrium geometry of the ground-state $\mathrm{OH}$ ( $R=1.830$ a.u.) [42], this basis set yielded the dipole moment of $1.789 \mathrm{D}$, which agrees reasonably well with the experimental value of $1.688 \mathrm{D}$ [42].

\section{RESULTS AND DISCUSSION}

In Figs. 1-4 we show our calculated spin-averaged DCS's (rotationally summed) for elastic $e^{-}-\mathrm{OH}$ scattering in the $2-500-\mathrm{eV}$ energy range. The $R$-matrix DCS's, calculated by Chen and Morgan [26] at the single incident energy of $1.58 \mathrm{eV}$, are shown in Fig. 1(a) to be compared with our results of $2 \mathrm{eV}$. Since no experimental or other theoretical results of DCS's for this target are available in the literature, we also use the existing calculated [45-49] and measured [50-53] results for elastic $e^{-}-\mathrm{H}_{2} \mathrm{O}$ collisions to compare with our data. A similar procedure has already been adopted in some previous studies for $\mathrm{CH}$ [6] and $\mathrm{SiH}$ [7] radicals. Thus we expect that it may provide some insight of the dynamics for elastic $e^{-}-\mathrm{OH}$ collision. It is interesting to see, in Fig. 1 (a), that there is a fairly good agreement between our calculated results at $2 \mathrm{eV}$ and the $R$-matrix DCS's at $1.58 \mathrm{eV}$ at 

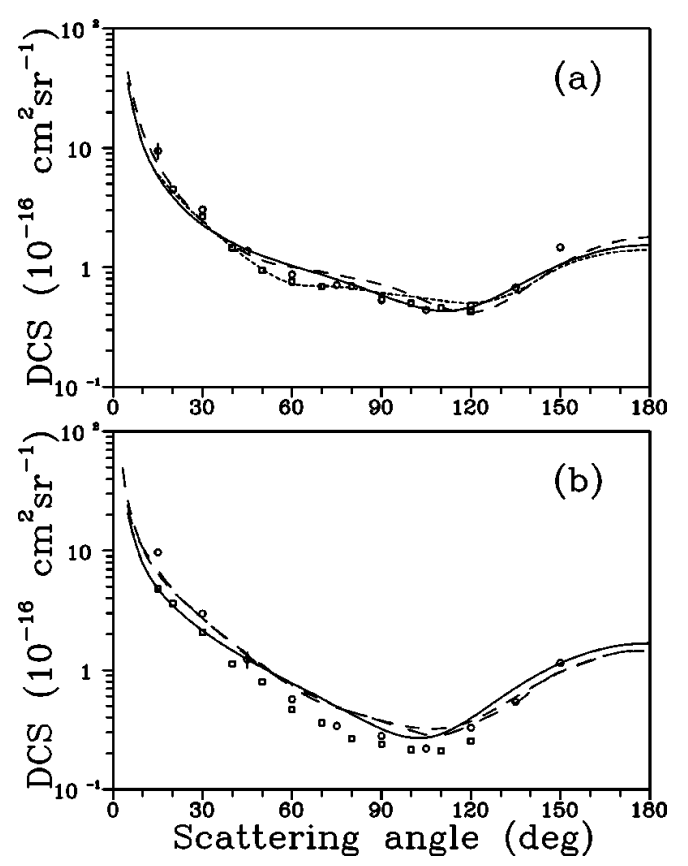

FIG. 2. Same as Fig. 1 except at (a) $10 \mathrm{eV}$ and (b) $20 \mathrm{eV}$. Open squares, experimental DCS's for $e^{-}-\mathrm{H}_{2} \mathrm{O}$ scattering of Danjo and Nishimura [50].

the intermediate and large scattering angles. However, the $R$-matrix results present a deep falloff at small scattering angles, due to the nonconvergent partial-wave expansions. In addition, one notes that at $2 \mathrm{eV}$, there is a good agreement between the present calculated results for $\mathrm{OH}$ and those calculated for $\mathrm{H}_{2} \mathrm{O}$, particularly at small scattering angles. It is
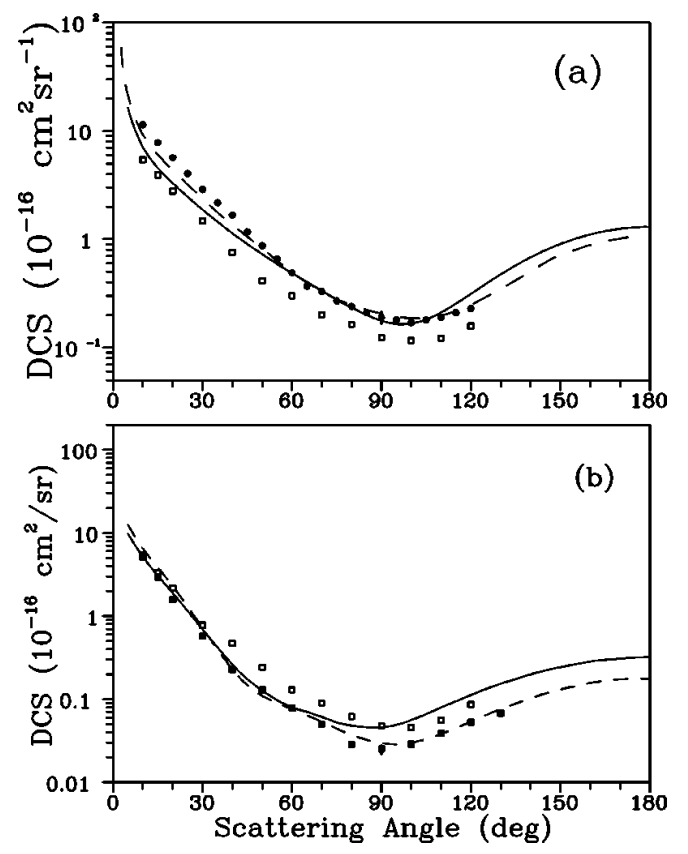

FIG. 3. Same as Fig. 1 except at (a) $30 \mathrm{eV}$ and (b) $100 \mathrm{eV}$. Full squares, experimental data for $e^{-}-\mathrm{H}_{2} \mathrm{O}$ scattering of Katase et al. [51]; dashed line, calculated results for $\mathrm{H}_{2} \mathrm{O}$ of Machado [49].
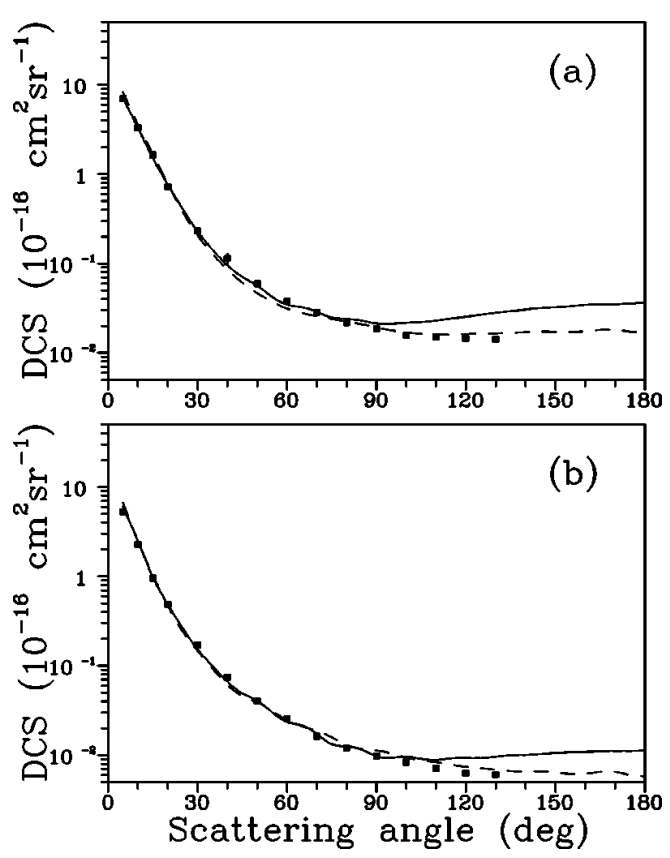

FIG. 4. Same as Fig. 3, except at (a) $300 \mathrm{eV}$ and (b) $500 \mathrm{eV}$.

well known that at such a low incident energy, the longrange interaction potentials of either permanent and/or induced natures are dominant in the collisional dynamics. The fact that the similarity of the permanent dipole of the targets, $1.68 \mathrm{D}$ for $\mathrm{OH}$ and $1.85 \mathrm{D}$ for $\mathrm{H}_{2} \mathrm{O}$ [42], may explain this good agreement, despite the larger difference in the average polarizability, 7.4 a.u. for $\mathrm{OH}$ and 10.5 a.u. for $\mathrm{H}_{2} \mathrm{O}$ [39]. It is due to the fact that polarization effects are less important for strongly polar targets. At higher incident energies, remarkable similarity between the calculated DCS's for electron scattering by $\mathrm{OH}$ and $\mathrm{H}_{2} \mathrm{O}$ are more apparent. This good agreement clearly indicates that the electron interaction with the heavier oxygen atom is dominant for both targets and the loss of one hydrogen atom in $\mathrm{OH}$ is not relevant. Also, the good agreement between the calculated and experimental DCS's for $e^{-}-\mathrm{H}_{2} \mathrm{O}$ scattering may provide some indications of the reliability of the present study.

In Figs. 5(a) and 5(b) we show our spin-averaged ICS's and MTCS's for elastic $e^{-}-\mathrm{OH}$ collisions, respectively, calculated in the $1-500-\mathrm{eV}$ range. Again, comparison is made with the calculated [45-49] and experimental [50-52] results for $e^{-}-\mathrm{H}_{2} \mathrm{O}$ scattering. In general, ICS's and MTCS's for elastic electron scattering by both targets agree well with each other, which again reinforces that the electron-oxygen atom interaction is dominant. Also, a broad enhancement centered at around $13 \mathrm{eV}$ is seen in both ICS's and MTCS's. In fact, this enhancement is a consequence of the occurrence of weak resonances in ${ }^{1,3} \Sigma$ and ${ }^{1,3} \Pi$ scattering channels. A similar resonance feature is also seen, at about the same energies, in $e^{-}-\mathrm{H}_{2} \mathrm{O}$ scattering.

In Fig. 6(a) we show our spin-averaged TCS's for $e^{-}-\mathrm{OH}$ collisions calculated in the $1-500-\mathrm{eV}$ range. The TCS's for this radical calculated by Joshipura et al. [5] using the additivity rule as well as calculated [54] and experimental [55-58] TCS's for $e^{-}-\mathrm{H}_{2} \mathrm{O}$ scattering available in the lit- 


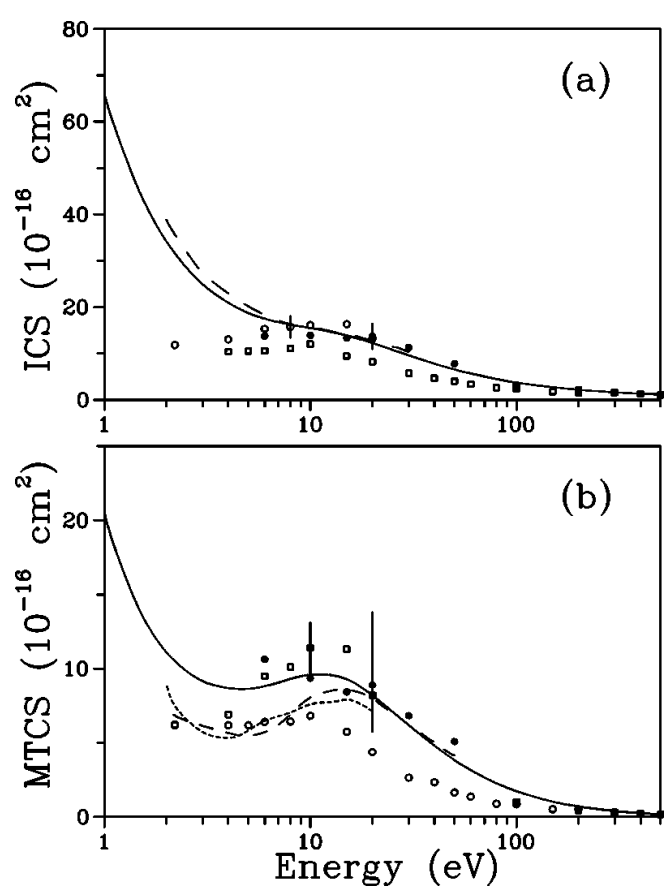

FIG. 5. (a) ICS's and (b) MTCS's for elastic $e^{-}-\mathrm{OH}$ scattering. Solid line, present rotationally summed results; the calculated results for $e^{-}-\mathrm{H}_{2} \mathrm{O}$ scattering are of: short-dashed line, Rescigno and Lengsfield [46]; dashed line, Machado et al. [47]; long-dashed line, Gianturco [45]. The experimental results for $e^{-}-\mathrm{H}_{2} \mathrm{O}$ scattering are of: open squares, Danjo and Nishimura [50]; open circles, Shyn and Cho [52]; full squares, Katase et al. [51]; full circles, Johnstone and Newell [53]. The quoted experimental uncertainties of the ICS's vary from $13 \%$ to $22 \%$ and those of the MTCS's are from $13 \%$ to $55 \%$. Representative error bars of some selected data are also shown.

erature are shown for comparison. In general, there is a good agreement between the present data and those of Joshipura et al. . However, the calculated TCS of Jain [54] for $e^{-}-\mathrm{H}_{2} \mathrm{O}$ scattering lie systematically above our data. On the other hand, there is a significant discrepancy among the experimental TCS's for $e^{-}-\mathrm{H}_{2} \mathrm{O}$ scattering, particularly at the low incident energies. It is seen that the present calculated data agree better with those of Sokolov and Sokolova [55] at low energies and with those of Sueoka et al. [56] at high incident energies. In Fig. 6(b) we show our spin-averaged TACS's for $e^{-}-\mathrm{OH}$ collisions calculated in the $10-500-\mathrm{eV}$ range. The TACS's calculated by Joshipura et al. [5] using the additivity

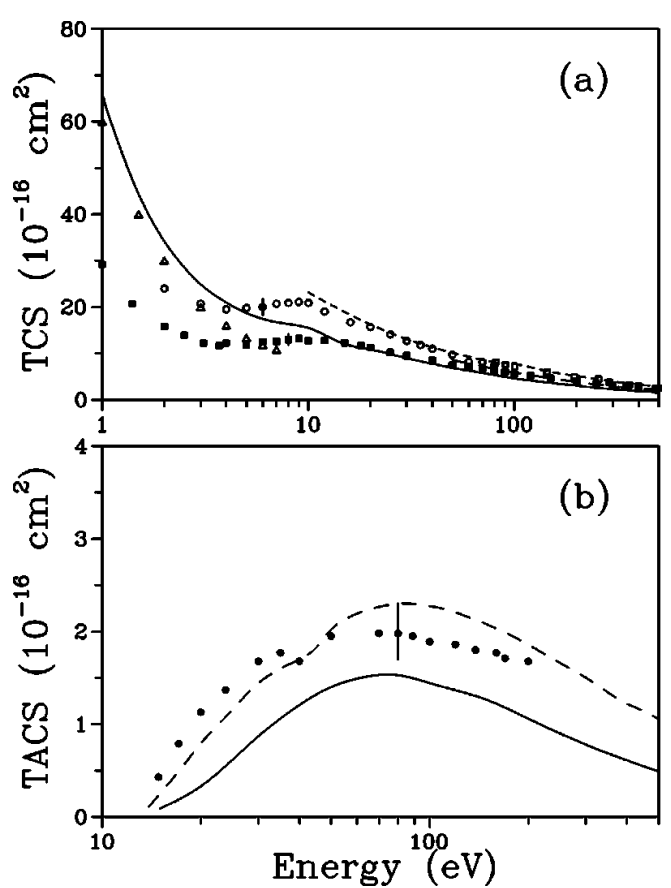

FIG. 6. (a) TCS's and (b) TACS's for $e^{-}-\mathrm{OH}$ scattering. Solid line, present results; dashed line, the calculated results for $e^{-}-\mathrm{OH}$ scattering of Joshipura et al. [5]; short-dashed line, the calculated results for $e^{-}-\mathrm{H}_{2} \mathrm{O}$ scattering of Jain [54]; full circles, the experimental TICS for $\mathrm{OH}$ of Tanovsky et al. [25]. The experimental TCS's for $e^{-}-\mathrm{H}_{2} \mathrm{O}$ scattering are of: open triangles, Sokolov and Sokolova [55]; full squares; Sueoka et al. [56]; open circles, Szmytkowski [57]; open squares, Zecca et al. [58]. The quoted experimental uncertainties of the TCS's vary from $1 \%$ to $10 \%$ and those of the TICS's are $15 \%$. Representative error bars of some selected data are also shown.

rule as well as the experimental TICS's of Tanovsky et al. [25] for this radical are also shown for comparison. In general, there is a good qualitative agreement among the calculated and experimental data. Quantitatively, the agreement between the present TACS's and the experimental TICS's is fair.

\section{ACKNOWLEDGMENTS}

This work was partially supported by the Brazilian agencies FAPESP, CNPq, and CAPES.
[1] S. Trajmar, D. F. Register, and A. Chutjian, Phys. Rep. 97, 219 (1983)

[2] J. Hahn and C. Junge, Z. Naturforsch. A 32, 190 (1977).

[3] W. Wang and N. D. Sze, Nature (London) 286, 589 (1980).

[4] K. N. Joshipura and M. Vinodkumar, Phys. Lett. A 224, 361 (1997).

[5] K. N. Joshipura, M. Vinodkumar, and P. M. Patel, J. Phys. B 34, 509 (2000).
[6] M.-T. Lee, M. F. Lima, A. M. C. Sobrinho, and I. Iga, J. Phys. B 35, 2437 (2002).

[7] M.-T. Lee, M. F. Lima, A. M. C. Sobrinho, and I. Iga, Phys. Rev. A 66, 062703 (2002).

[8] M.-T. Lee, I. Iga, L. M. Brescansin, L. E. Machado, and F. B. C. Machado, Phys. Rev. A 66, 012720 (2002).

[9] S. C. Brown, Electron-Molecule Scattering (Wiley, New York, 1979). 
[10] R. K. Jane, in Atomic and Plasma-Material Interaction Processes in Controlled Thermonuclear Fusion, edited by R. K. Janev and H. W. Drawin (Elsevier, Amsterdam, 1993), p. 27.

[11] J. J. Perry, Y. H. Kim, J. L. Fox, and H. S. Porter, J. Geophys. Res. 104, 16541 (1999).

[12] G. Heit, A. Neuner, P. Y. Saugy, and A. M. Braun, J. Phys. Chem. A 102, 5551 (1998)

[13] D. J. Creasey, G. E. Evans, D. E. Heard, and J. D. Lee, J. Geophys. Res., [Atmos.] 108, 4475 (2003).

[14] M. Vrtacnik and K. Voda, Chemosphere 52, 1689 (2003).

[15] X. R. Ren, H. Harder, M. Martines, R. L. Lesher, A. Oliger, and J. B. Simpas, W. H. Brune, J. J. Schwab, K. L. Demerjian, Y. He, X. L. Zhou, and H. G. Gao, Atmos. Environ. 37, 3639 (2003).

[16] R. Atkinson, Atmos. Chem. Phys. 3, 2233 (2003).

[17] K. E. S. Ford, D. A. Neufeld, P. F. Goldsmith, and G. J. Melnick, Astrophys. J. 589, 430 (2003).

[18] J. P. Morgenthaler, W. M. Harris, F. L. Roesler, F. Scherb, C. M. Anderson, N. E. Doane, and R. J. Oliversen, Earth, Moon, Planets 90(1-4), 77 (2002).

[19] D. A. Neufeld, J. R. Stauffer, E. A. Bergin, S. C. Kleiner, B. M. Patten, Z. Wang, M. L. N. Ashby, G. Chin, N. R. Erickson, P. F. Goldsmith, M. Harwit, J. E. Howe, D. G. Koch, R. Plume, R. Schieder, R. L. Snell, V. Tolls, G. Winnewisser, Y. F. Zhang, and G. J. Melnick, Astrophys. J. Lett. 539, L151 (2000).

[20] E. Fernandes, S. A. Toste, J. L. F. C. Lima, and S. J. Reis, Free Radic Biol. Med. 35, 1008 (2003).

[21] S. S. Leonard, C. Xia, B. H. Jiang, B. Stinefelt, H. Klandorf, G. K. Harris, and X. L. Shi, Biochem. Biophys. Res. Commun. 309, 1017 (2003).

[22] I. P. Kumar, S. V. S. Rana, N. Samanta, and H. C. Goel, J. Pharm. Pharmacol. 55, 1267 (2003).

[23] H. B. An, J. Xie, J. Q. Zhao, and Z. S. Li, Free Radical Res. 37, 1107 (2003).

[24] A. Katase, K. Ishibashi, Y. Matsumoto, T. Sakae, S. Maezono, E. Murakami, K. Watanabe, and H. Maki, J. Phys. B 19, 2715 (1986).

[25] V. Tanovsky, H. Deutsch, and K. Becker, J. Chem. Phys. 109, 932 (1998).

[26] X. Chen and L. A. Morgan, J. Phys. B 30, 3709 (1997).

[27] R. R. Lucchese, G. Raseev, and V. McKoy, Phys. Rev. A 25, 2572 (1982).

[28] L. Mu-Tao, L. M. Brescansin, M. A. P. Lima, L. E. Machado, and E. P. Leal, J. Phys. B 23, 4331 (1990).

[29] A. W. Fliflet and V. McKoy, Phys. Rev. A 21, 1863 (1980).

[30] M.-T. Lee and V. McKoy, Phys. Rev. A 28, 697 (1983).
[31] M.-T. Lee, S. Michelin, L. E. Machado, and L. M. Brescansin, J. Phys. B 26, L203 (1993).

[32] M.-T. Lee, I. Iga, L. E. Machado, and L. M. Brescansin, Phys. Rev. A 62, 062710 (2000).

[33] M.-T. Lee and I. Iga, J. Phys. B 32, 453 (1999).

[34] I. Iga, M. G. P. Homem, K. T. Mazon, and M.-T. Lee, J. Phys. B 32, 4373 (1999).

[35] S. E. Michelin, T. Kroin, I. Iga, M. G. P. Homem, and M.-T. Lee, J. Phys. B 33, 3293 (2000).

[36] N. T. Padial and D. W. Norcross, Phys. Rev. A 29, 1742 (1984).

[37] A. Faure, J. D. Gorfinkiel, and J. Tennyson, J. Phys. B 37, 801 (2004).

[38] C. S. Trevisan, A. E. Oral, and T. N. Rescigno, Phys. Rev. A 68, 062707 (2003).

[39] C. Winstead and V. McKoy, J. Chem. Phys. 116, 1380 (2002).

[40] G. Staszewska, D. W. Schwenke, and D. G. Truhlar, Phys. Rev. A 29, 3078 (1984).

[41] A. Jain and K. L. Baluja, Phys. Rev. A 45, 202 (1992).

[42] D. R. Lide, CRC Handbook of Chemistry and Physics (Chemical Rubber Company, Boca Raton, FL, 1993).

[43] T. H. Dunning, J. Chem. Phys. 55, 716 (1971).

[44] S. Huzinaga, J. Chem. Phys. 42, 1293 (1965).

[45] F. A. Gianturco, J. Phys. B 24, 3837 (1991).

[46] T. Rescigno and B. M. Lengsfield, Z. Phys. D: At., Mol. Clusters 24, 117 (1992).

[47] L. E. Machado, M.-T. Lee, L. M. Brescansin, M. A. P. Lima, and V. McKoy, J. Phys. B 28, 476 (1995).

[48] M. T. N. Varella, M. H. F. Bettega, M. A. P. Lima, and L. G. Ferreira, J. Chem. Phys. 111, 6369 (1999).

[49] L. E. Machado (private communication).

[50] A. Danjo and H. Nishimura, J. Phys. Soc. Jpn. 54, 1224 (1985).

[51] A. Katase, K. Ishibashi, Y. Matsumoto, T. Sakae, S. Maezono, E. Murakami, K. Watanabe, and H. Maki, J. Phys. B 19, 2715 (1986).

[52] T. W. Shyn and S. Y. Cho, Phys. Rev. A 36, 5138 (1987).

[53] W. M. Johnstone and W. R. Newell, J. Phys. B 24, 3663 (1991).

[54] A. Jain, J. Phys. B 21, 905 (1988).

[55] V. F. Sokolov and Y. A. Sokolova, Sov. Tech. Phys. Lett. 7, 268 (1981).

[56] O. Sueoka, S. Mori, and Y. Katayama, J. Phys. B 19, L373 (1986).

[57] Cz. Szmytkowski, Chem. Phys. Lett. 136, 363 (1987).

[58] A. Zecca, G. Karwasz, S. Oss, R. Grisenti, and R. S. Brusa, J. Phys. B 20, L133 (1987). 